Ann. Biol. anim. Bioch. Biophys., I966, 6 (3), 35I-37I.

\title{
LES ACIDES GRAS TRANS DU BEURRE
}

\author{
II. -- CONTRIBUTION A L'T́tTUDE DE LEUR ORIGINE
}

Simonne KUZDZAL-SAVOIE, Janine RAYMOND, W. KUZDZAL et Janine PETIT

Station centrale de Recherches laitières et de Technologie des Produits animaux, Centre national de Recherches zootechniques, 78-Jouy-en-Josas

\section{SOMMAIRE}

Les acides gras " trans " du beurre préexistent dans les lipides du lait. Leur origine est essentiellement endogène : ils prennent naissance au cours d'un phénomène d'hydrogénation qui existe en particulier, au niveau du rumen et qui porte sur les acides polyinsaturés de la ration.

Les résultats obtenus ont permis d'établir l'existence d'une relation directe entre le taux d'acide linolénique de la ration et le taux des acides gras trans des lipides du lait.

Mais le site précis de formation de ces acides trans, et la nature des composés intermédiaires entre l'acide linolénique et l'acide trans-octadécène-I I-oíque (acide vaccénique), par exemple, restent encore assez mal définis.

\section{I. - INTRODUCTION}

L'étude des acides gras du beurre est un sujet de recherches toujours actuel. En ig62, Magidman et al. détectaient 64 acides gras dans les lipides du lait de vache. Les travaux récents de IVERson et al. (I965) portent ce nombre à 84 .

Dans une précédente publication (KUZDZAL-SAvole et RAYMOND, I965) nous avons rappelé les connaissances actuelles concernant les acides gras trans du beurre. Nous avons en outre proposé une méthode d'isolement et de dosage pondéral des acides gras trans du beurre basée sur les récents progrès de l'analyse chromatographique. Cette méthođe a été appliquée à l'étude de quelques échantillons de beurre fabriqués à différentes périodes de l'année.

Les acides gras trans du beurre préexistent dans les lipides du lait. Leur origine est essentiellement endogène car les aliments naturels habituellement distribués aux vaches laitières ne contiennent qu'une quantité négligeable d'acides trans (Brou- 
WER et JONKER-SCHEFFENER, I946; HARTMAN et al., I954 et x956). On peut retenir de diverses études (Brouwer et JONKER-SCHEFFEnER, I946; KUZIZAL-SAvoIE, I964) que le taux des isomères trans dans le lait est d'autant plus élevé que la ration alimentaire des vaches laitières comporte une plus grande quantité d'herbe jeune (printemps, automne). L,es lipides de l'herbe jeune contiennent une proportion élevée d'acides polyinsaturés: linoléique é surtout linolénique. Ces acides polyinsaturés n'apparaissent que très partiellement dans le lait par suite de l'existence au niveau du rumen d'un phénomène d'hydrogénation, - dont les micro-organismes sont responsables -, qui les transforment en acides plus saturés: monoènes et acide stéarique. L,es proportions de ces acides s'élèvent dans les lipides du lait lorsque de l'herbe jeune est introduite dans la ration (cf. KuzDzAL-SAvoIE, I964). Parmi les monoènes du lait on compte alors tune proportion notable d'acides trans.

Il a été établi que les acides gras trans des lipides du lait prennent naissance au cours de 1'hydrogénation dans le rumen (hydrogénation biologique). Des expériences in vivo et surtout in vitro, ont été effectuées en vue de préciser la nature des acides gras formés (produits intermédiaires et produits finaux) au cours de l'hydrogénation biologique portant sur les acides oléique, linoléique et linolénique (SHorLAND et $a l .$, I957; WARD et al., I964). Les isomères formés au cours de 1'hydrogénation catalytique portant sur ces mêmes acides ont fait également l'objet de nombreuses investigations. Les recherches de DuTTON (I963) et de SREENIVASAN et al. (I963) sur l'hydrogénation de l'acide linolénique nous intéressent en premier lieu. En effet l'acide linolénique est l'acide majeur des lipides de l'herbe et des fourrages; sa proportion dans les lipides de la ration varie done fortement au cours de l'année alors que le taux d'acide linoléique varie relativement peu (l'acide linoléique est l'acide gras majeur des aliments concentrés à base de céréales souvent distribués l'hiver) et que le taux d'acide oléique reste toujours faible (cf. KuZDZAI-SAvOIE, I964).

Selon l'étude récente de WARD et al. (Ig64), 1'hydrogénation biologique s'exerçant sur l'acide linolénique conduit aux produits terminaux suivants : acide stéarique et acides monoènes comportant I 8 atomes de carbone, de configuration trans principalement. Selon WARD et al., la double liaison des monoènes trans serait placée préférentiellement sur les atomes de carbone I3 et I4 et secondairement sur les atomes voisins. Cependant si une hydrogénation de 3 ou 4 heures portant sur l'acide linolénique (hydrogénation obtenue grâce à l'action des micro-organismes dans l'enceinte d'un rumen artificiel) conduit aux produits terminaux dont nous venons de parler, une hydrogénation limitée à 20 minutes a permis de mettre en évidence des produits intermédiaires étudiés par WARD et al. (Ig64). Ces produits intermédiaires sont composés essentiellement de deux acides diènes à $I 8$ atomes de carbone, l'un non conjugué à doubles liaisons éloignées et l'autre conjugué ; tous deux de configuration cis-cis. Cependant ces résultats ne nous permettent d'expliquer que partiellement l'origine des acides trans du beurre puisque l'acide vaccénique qui en constitue la majeure partie possède une double liaison sur le carbone II, et que l'acide diène conjugué signalé dans le beurre possède une configuration cis-trans ou trans-trans (SMITH et JACK, I954; НЕRB et al., Ig62).

Sans doute d'autres phénomènes que la simple hydrogénation portant sur l'acide linolénique doivent être mis en cause. Parmi ceux-ci nous citerons la participation directe des acides monoènes à $\mathrm{I} 8$ atomes de carbone des lipides protoplasmiques des micro-organismes du rumen ou du tractus digestif plus éloigné. Les travaux de KATZ 
e $\iota$ KEENFY (1963) ont montré en particulier que, dans la moitié des cas, la double liaison de ces monoènes est placée sur le carbone I I et, si l'on considère les seuls trans monoènes des lipides de ces micro-organismes, dans go p. Ioo des cas, la double liaison est sur le carbone Ir.

On sait aussi que l'hydrogénation se poursuit dans les parties inférieures du tractus digestif (WARD et al., I964). Elle porte alors préférentiellement sur 1'acide linoléique et on assiste à une nouvelle formation d'acides monoènes trans dont la position de la double liaison n'a pas été déterminée.

Cependant si l'apport d'acide linolénique alimentaire est le phénomène qui détermine un accroissement d'acides trans (trans-octadécène- I I-oïque) dans les lipides du lait, l'hydrogénation portant sur l'acide linolénique, au niveau du rumen, doit être le phénomène central en cause. L'hypothèse d'une isomérisation de la double liaison des monoènes trans sous l'action des micro-organismes peut être émise, par analogie avec un phénomène de même nature déjà démontré ( $O^{\prime} L_{E A R Y}$, I959). L'hypothèse d'une résorption des seuls isomères trans-II est, par contre, difficilement concevable.

L'origine exacte des acides gras trans du beurre est donc encore assez mal établie, et plusieurs voies expérimentales peuvent être suivies en vue de préciser cette origine.

En premier lieu il convient de vérifier si l'apport supplémentaire d'acide linolénique dans la ration des vaches laitières entraîne un accroissement du taux des acides trans des lipides du lait, et si l'absence d'acide linolénique dans la ration entraîne leur disparition complète, — ou seulement une diminution -, des acides trans du lait (monoènes à 18 atomes de carbone). Il est de plus nécessaire de vérifier que les acides trans dont la formation peut être provoquée par l'apport d'acide linolénique dans la ration possèdent préférentiellement la double liaison trans en position II.

Une fois ces points établis, le site de formation des acides trans monoènes du lait, et la nature des composés intermédiaires entre l'acide linolénique et l'acide transI I-otadécènoïque en particulier, devraient pouvoir être précisés par des analyses comparables à celles présentées par WARD et al. (I964), mais comportant toujours l'isolement des acides cis et des acides trans, ainsi que la détermination de 1'emplacement des doubles liaisons.

La présente étude se rapporte au premier point de ce programme, à savoir l'établissement d'une relation directe entre l'acide linolénique de la ration et les acides gras trans des lipides du lait.

\section{II. - PARTIE EXPÉRIMENTALE}

\section{A - Le troupeau expérimental - Le régime alimentaire — Les prélèvements de lait}

Trois vaches de race Française Frisonne Pie Noire $(53 \mathrm{H}$, $195 \mathrm{H}$ et gor $3 \mathrm{H}$ ) appartenant au troupeau du Centre national de Recherches zootechniques sont retenues pour l'expérimentation. Elles reçoivent une alimentation à base de foin, de betteraves, d'ensilage et de concentré. Un supplément alimentaire, sous forme de tourteau gras de lin leur est distribué, à raison de I à $3 \mathrm{~kg}$ par vache et par jour selon un protocole expérimental décrit à la partie inférieure des figures i et 2 . La période expérimentale est de deux semaines. Des prélèvements sont effectués régulièrement pendant la période expérimentale et pendant les périodes pré- et post-expérimentales, sur le lait des vaches 
soumises à l'expérimentation. Un lait de mélange, pondéré en fonction des quantités de lait produites par chaque vache, et représentatif d'une journée est ensuite constitué. Dix prélèvements sont effectués du 17 février au 13 mars 1965.
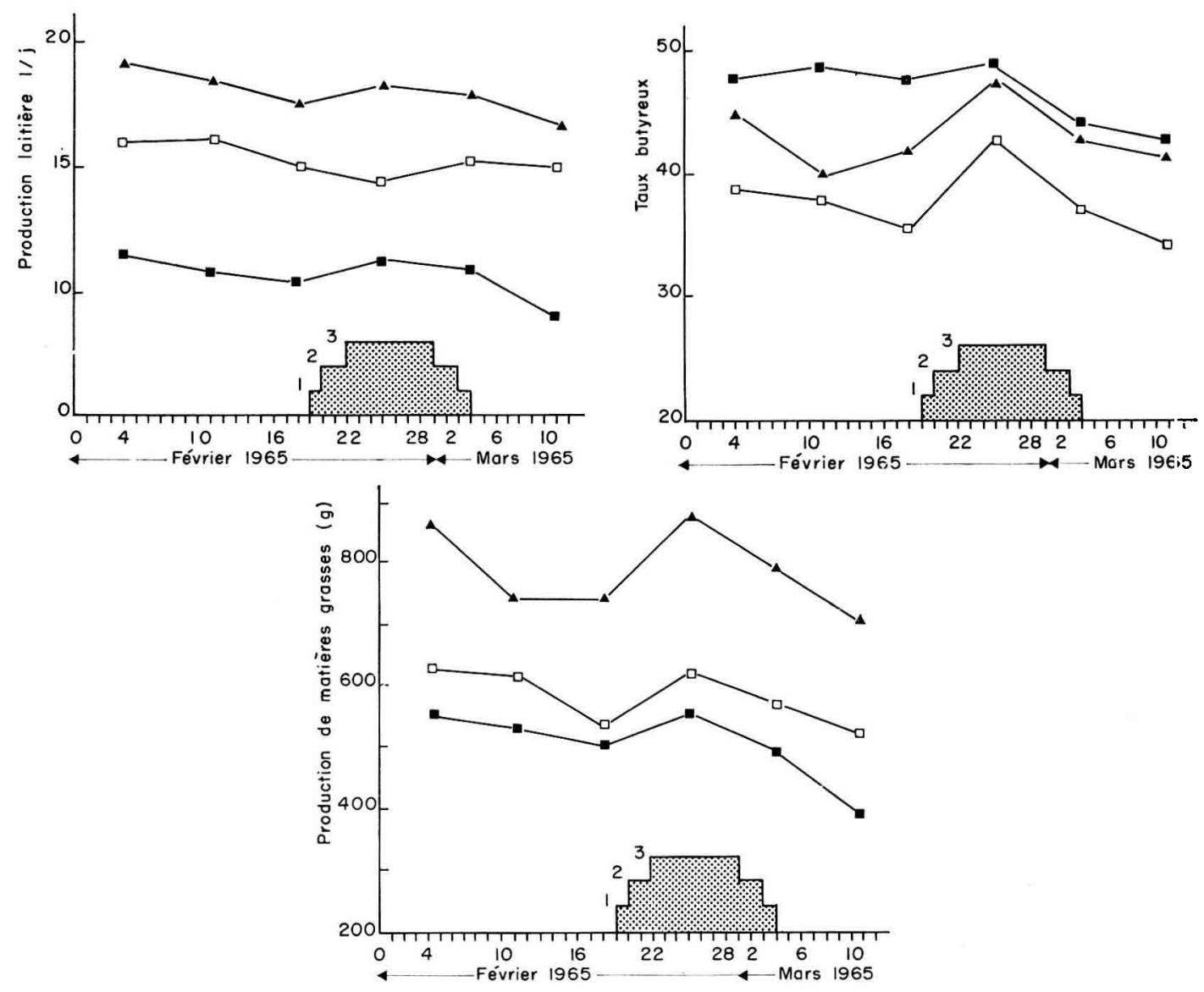

- $18: 2$ (linoléique)

O $18: 3$

$18: 2$ (conj. UV)

$18:$ (non linoéique)

03 tourteau de $\operatorname{lin}(\mathrm{kg})$

Vaches : $53 \wedge$, I95 $\square, 9013$

FIG. I. - Variations de la quantité journalière de lait produit par les trois vaches du troupeau expérimental (graphique supérieur gauche), du taux butyreux de ce lait (graphique supérieur droit) et de la quantitéjournaliève de matière grasse sécrélée (graphique inférieur) pendant la période du $1^{\mathrm{er}}$ février au 15 mars 1965 (moyennes hebdomadaires).

\section{B. Analyse du tourteau de lin. Analyse des lipides du lait}

a) Le tourteau de lin.

On détermine l'extrait sec, le taux d'huile et la composition des acides gras de cette huile par l'analyse chromatographique des esters méthyliques préparés par transestérification (cf. KUzDZALSAVOIE et KUZDZAL, 1962).

b) Les lipides du lait.

Le lait est écrémé. La crème est transformée en beurre dans un mixer tournant à I 2 ooo tours par minute. Le beurre est ensuite chauffé au bain-marie à $50^{\circ} \mathrm{C}$ et centrifugé. Sur l'huile obtenue on détermine le taux d'acides diènes conjugués selon la méthode décrite par BRICE et SwaIN (1945) 
et précédemment utilisée (cf. KuzdzAL-SAvoIE et KuzDzaL, I96r). On prépare à partir de l'huile de beurre, les esters méthyliques par transestérification (KuzDzaL-SAvoIE et KUzDzAL, I962) et on soumet les esters à l'analyse par chromatographie en phase gazeuse. Par ailleurs, les esters méthyliques des acides gras trans sont isolés, dosés et identifiés selon le mode opératoire décrit antérieurement

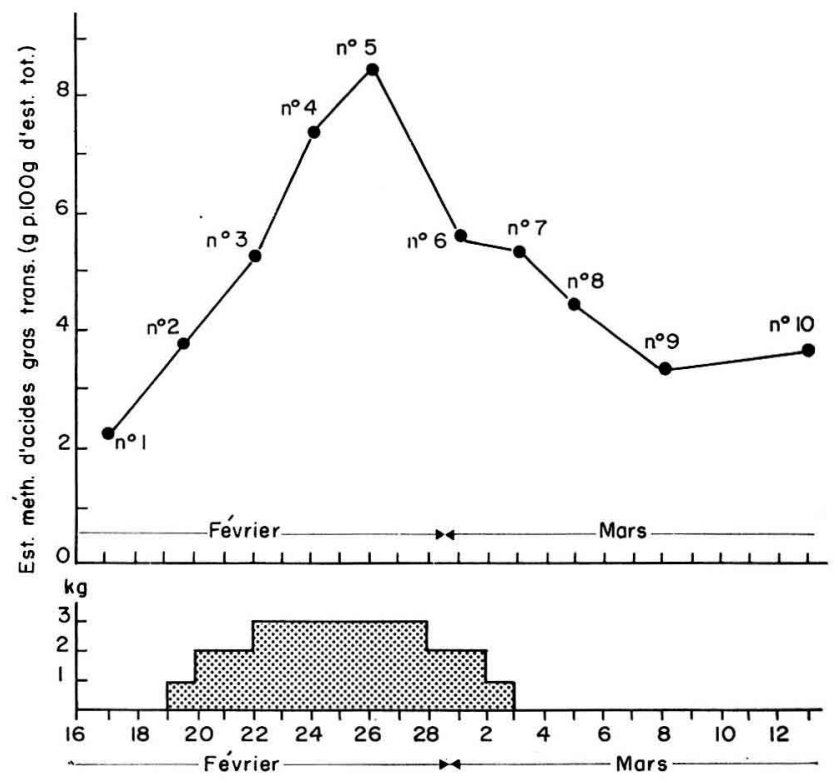

2 tourteau de lin

FIG. 2. - Variation de la teneur en acides gras trans du beurre, au cours de l'expérimentation (teneur exprimée en g p. roo g d'esters méthyliques déposés).

(KuzDzal-Savole et RAYmond, I965). Les esters d'acides saturés et insaturés cis obtenus en groupes distincts au cours de cette analyse sont également étudiés par chromatographie en phase gazeuse. L'analyse directe des esters méthyliques totaux du beurre, par chromatographie sur couche mince de silicagel imprégné de nitrate d'argent est effectuée selon PALLoTA et al. (I965).

\section{III. — RÉSULTATS}

\section{A - Résultats de l'analyse du tourteau de lin}

Les résultats se rapportant à l'analyse du tourteau de lin et de l'huile extraite de ce tourteau sont portés dans le tableau I. L'huile contient une proportion élevée d'acide linolénique voisine de $60 \mathrm{p}$. Ioo des acides totaux.

Connaissant la proportion d'acide linolénique dans l'huile de lin, le taux d'huile dans le tourteau et la quantité de tourteau distribuée à chaque vache, chaque jour, il est possible de connaître la quantité d'acide linolénique ajoutée à la ration de base. Ainsi les vaches reçurent environ $\mathrm{I}_{2} 5,255$ ou $380 \mathrm{~g}$ d'acide linolénique pour $\mathrm{I}, 2$ ou $3 \mathrm{~kg}$ de tourteau gras de lin. 


\section{$\mathrm{B}$ - Résultats de l'analyse des lipides du lait}

a) Production de matiere grasse.

Le taux butyreux du lait, les quantités de lait produites par les vaches en expérimentation et les quantités de matière grasse sécrétée sont indiquées sur la figure $\mathrm{I}$.

TABLEAU I

Analyse de l'huile extraite du tourteau de lin

Tourteau : Extrait sec : 91,5 p. 100 ,

Gras/sec : 24,0 p. 100

Résultats exprimés en p. 100 des esters méthyliques totaux

\begin{tabular}{c|c|c|c|c}
\hline $\begin{array}{c}\text { Acide } \\
\text { palmitique } \\
16: 0\end{array}$ & $\begin{array}{c}\text { Acide } \\
\text { stéarique } \\
18: 0\end{array}$ & $\begin{array}{c}\text { Acide } \\
\text { oléique } \\
18: 1\end{array}$ & $\begin{array}{c}\text { Acide } \\
\text { lnoléique } \\
18: \pm\end{array}$ & $\begin{array}{c}\text { Acide } \\
\text { linolénique } \\
18: 3\end{array}$ \\
\hline 5,8 & 3,1 & 15,0 & 17,0 & 59,1 \\
\hline
\end{tabular}

On constate que la période expérimentale correspond à une élévation faible mais notable de la quantité de matière grasse sécrétée. Cette élévation temporaire s'aligne sur la distribution de tourteau de lin.

b) Les acides gras trans.

Les résultats concernant l'analyse par chromatographie sur colonne de silicagel imprégné de nitrate d'argent, et conduisant au fractionnement des esters méthyliques des acides totaux en esters d" "acides saturés ", d' " acides insaturés trans" (monoènes trans) et "insaturés cis", sont portés dans le tableau 2. Sur la figure 2 on présente la variation du taux des acides trans dans les échantillons de beurre préparés à partir du lait prélevé, et sur la figure 3, une analyse par chromatographie en couche mince sur silicagel imprégné de nitrate d'argent des esters méthyliques totaux de l'échantillon de beurre $\mathrm{n}^{0} 6$. On observe, très bien détaché, le "feston " d'acides trans à la partie supérieure de la figure. On observe une nette augmentation du taux des acides trans au cours de l'expérimentation : il est de 2 à 3 p. Ioo hors expérimentatioül, et atteint 8,5 p. Ioo pendant la période de distribution maximale de tourteau delin soit une valeur triple de la valeur de départ.

Le taux d'acides trans le plus élevé atteint au cours de la période expérimentale $(8,5$ p. IOo) est voisin du taux $(7,5$ p. IOo) qui a été trouvé par la même méthode d'analyse; dans un beurre industriel fabriqué en mai I964, c'est-à-dire pendant une période de vie au pâturage et de consommation d'herbe jeune par les vaches laitières (Kuzdzal-SAvoie et Raymond, I965). 
L'observation du tableau 2. montre en outre que la proportion d'acides insaturés cis s'est élevée sensiblement au cours de l'expérimentation. Ainsi la diminution d'acides saturés est compensée par une augmentation d'acides insaturés-trans et insaturés-cis. Cette augmentation est du même ordre pour les insaturés-trans et les insaturés-cis.

\section{TABLEAU 2}

Fractionnement des esters méthyliques des acides gras du beurre sur colonne d'acide silicique imprégné de nitrate d'argent

\begin{tabular}{|c|c|c|c|c|c|}
\hline $\begin{array}{c}\text { No des } \\
\text { échantillons }\end{array}$ & $\begin{array}{c}\text { Date du } \\
\text { [rélèvement }\end{array}$ & $\begin{array}{c}\text { Esters } \\
\text { saturés (1) }\end{array}$ & $\begin{array}{l}\text { Esters } \\
\text { insaturés-lrans (1) }\end{array}$ & $\begin{array}{c}\text { Esters } \\
\text { insaturés-cis (1) }\end{array}$ & $\begin{array}{l}\text { Pourcentages } \\
\text { récupérés (2) }\end{array}$ \\
\hline 1 & $17-2-65$ & 56,6 & 2,3 & 18,5 & 77,4 \\
\hline 2 & $18-2-65$ & 55,4 & - & 22,5 & - \\
\hline 3 & $2-2-2-65$ & 55,1 & 5,3 & 24,1 & 84,5 \\
\hline 4 & $2 / 4-2-65$ & 50,3 & 7,4 & 22,9 & 80,6 \\
\hline 5 & $26-2-65$ & 67,6 & 8,5 & 21,3 & 80,4 \\
\hline 6 & $1-3-65$ & 46,3 & 5,6 & 25,5 & $77,4_{4}$ \\
\hline 7 & $3-3-65$ & 47,7 & 5,4 & 23,8 & 76,9 \\
\hline 8 & $5-3-65$ & 50,8 & 1,5 & 24,3 & 79,6 \\
\hline g & $8-3-65$ & 52,8 & 3,4 & 23,3 & 79,5 \\
\hline 10 & $13-3-6-65$ & 53,5 & 3,7 & 22,1 & 79,6 \\
\hline
\end{tabular}

(1) Exprimés en g p. $100 \mathrm{~g}$ d'esters méthyliques totaux mis au départ. Valeurs moyennes obtenues généralement à partir de trois fractionnements.

(2) Ce pourcentage faible s'explique en grande partie par la perte des esters méthyliques à bas poids moléculaire et par la non-élution des esters d'acides polyinsaturés.

Les groupes d'acides (saturés, insaturés-trans et insaturés-cis) obtenus au cours du fractionnement sur colonne de silicagel imprégné de nitrate d'argent ont tous été étudiés par chromatographie en phase gazeuse. L'observation de l'ensemble des chromatogrammes révèle des images très semblables pour les groupes correspondants.

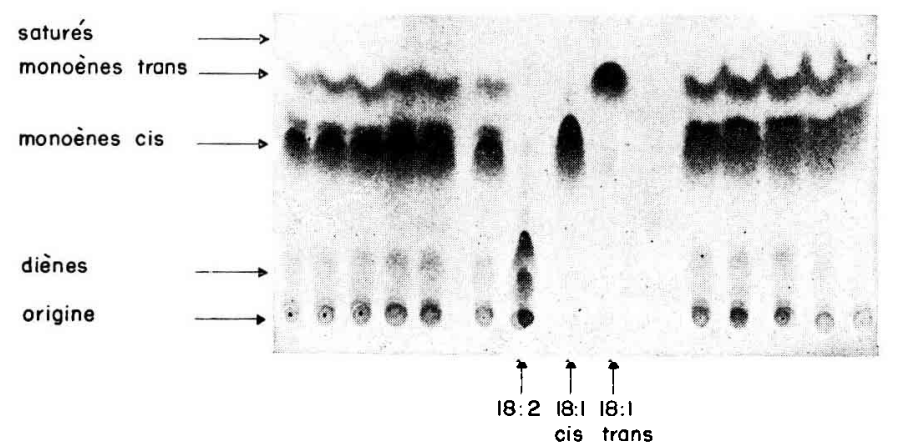

FIG. 3. - Chromatographie en couche mince sur silicagel imprégné de nitrate d'argent des esters méthyliques totaux du beurre $n^{0} 6$.

Conditions expérimentales : selon Pallotra et al. (1965): Silicagel-nitrate d'argent-eau (45/1 5/90). Épaisseur des plaques : $0,3 \mathrm{~mm}$. Solvant : benzène-éther de pétrole $(80 / 20)$. Révélateur : vapeurs d'iode et solution d'acide phosphomolybdique à 20 p. Ioo dans l'éthanol. 
On retrouve 1'aspect déjà décrit dans l'étude précédente (KUZDZAL-SAvoIE et RAYMOND, I 965) : les monoènes à I5, I6 et I7 atomes de carbone toujours présents dans les acides trans, dans lesquels se retrouvent généralement une fraction mineure d'acides saturés à $\mathrm{I} 2$ et $\mathrm{I} 4$ atomes de carbone (fig. 4). On observe régulièrement sur $1 \mathrm{a}$ partie descendante du pic I8:I un épaulement déjà signalé dans l'étude précédente.

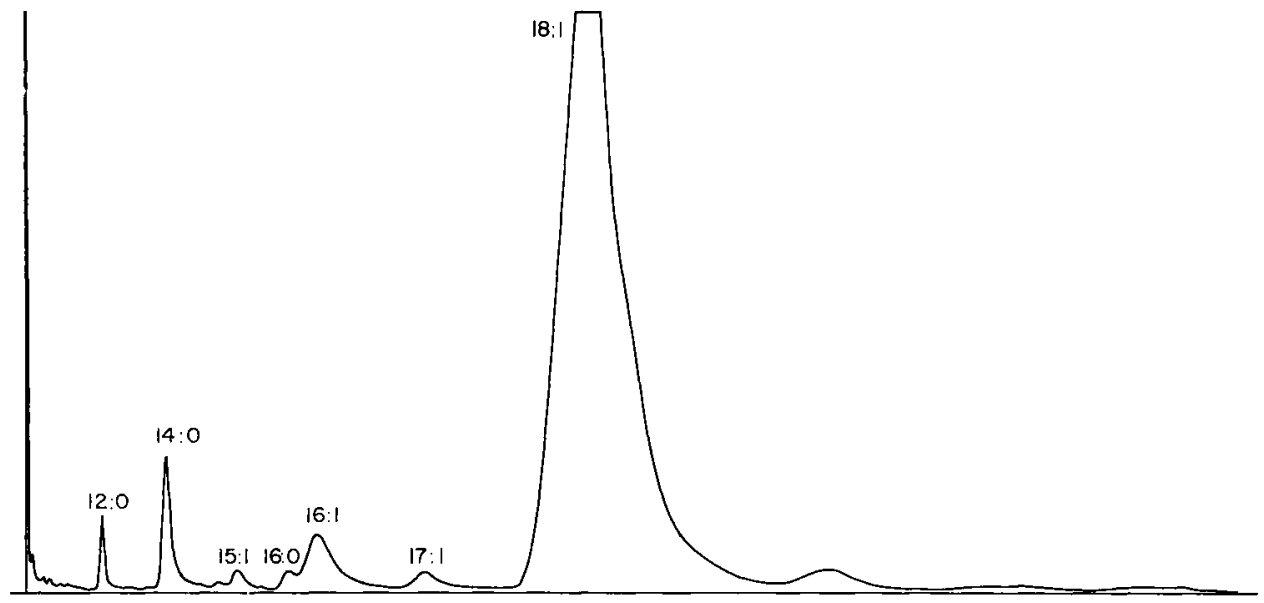

FIG. 4. - Analyse par chromatographie en phase gaseuse $d u$ groupe "esters d'acides trans "du beure $n^{\circ} j$ (colonne $H$ )

Conditions de l'analyse : Aerograph $\mathrm{IyTi} 600$ (ionisation de flamme).

Colonne : $3,048 \mathrm{~m}$ (10 pieds anglais) de long et $0,3^{2} \mathrm{~cm}$ (1 $/ 8$ de pouce anglais) de diamètre.

Phase : DLGS à Io p. $100(\mathrm{p} / \mathrm{p})$ sur chromosorb W lavé à l'acide.

Température : $180^{\circ} \mathrm{C}$ environ.

Gaz vecteur : azote; débit : 25 à $30 \mathrm{ml} / \mathrm{minute}$.

Le groupe des acides insaturés-cis est caractérisé par la présence en proportions relativement élevées des acides monoènes à I4, I 6 et I 7 atomes de carbone et par l'absence d'épattement sur la partie descendante du pic I8:I.

On a calculé, à partir des différents chromatogrammes, les proportions d'acides mineurs (monoènes trans?) évaluées d'abord en fonction des acides trans totaux puis en fonction des acides totaux du beurre.

On a de la même façon, calculé les proportions d'acides monoènes cis mineurs en fonction des acides cis monoènes totaux puis en fonction des acides totaux du beurre.

Énfin on a évalué les proportions des différents acides saturés majeurs en fonction des acides totaux du beurre. 'Tous ces résultats sont portés dans le tableau 3 (résultats se rapportant à l'analyse chromatographique sur colonne de silicagel imprégné de nitrate d'argent).

On considère dans ces calculs, mais ceci est assez approximatif, que la perte constatée après passage des esters méthyliques totaux sur colonne d'acide silicique imprégné de nitrate d'argent correspond à la perte des esters méthyliques des acides à bas poids moléculaire jusqu'à $\mathrm{C}_{12}$ (par suite des multiples opérations d'évaporation de solvant) et à la perte des esters d'acides polyinsaturés (non élués).

Ainsi les résultats obtenus au cours de l'analyse des groupes d'esters recueillis après passage sur colonne doivent correspondre approximativement aux résultats 
obtenus par l'analyse directe par chromatographie en phase gazeuse des esters méthyliques totaux du beurre.

c) Les acides gras totanx.

Au cours de la période qui s'étend du I 5 février au 20 mars I965, les proportions relatives des acides gras du lait de mélange obtenu à partir du lait produit par les trois vaches en expérimentation ont varié. Ces proportions relatives sont indiquées pour les dix échantillons de beurre préparés au cours de l'expérimentation dans le tableau 3.

Dans ce tableau se trouvent également les résultats obtenus lors de l'analyse des groupes d'esters résultant du fractionnement sur colonne de silicagel imprégné de nitrate d'argent.

Le recoupement des deux méthodes n'est pas parfait ; cependant il est suffisamment bon pour démontrer que le fractionnement sur colonne de silicagel imprégné de nitrate d'argent n'altère pas les proportions initales des acides gras et permet donc, par exemple, une évaluation relativement exacte des proportions relatives des acides cis et trans (?) monoènes mineurs.

Dans la période pré-expérimentale, la matière grasse du lait présente une composition caractéristique d'une matière grasse de lait d'hiver : taux élevés des acides à 14 et I 6 atomes de carbone et taux faibles des acides stéarique et oléique. L'apport d'acide linolénique dans la ration entraîne une augmentation de la proportion d'acide monoène à $\mathrm{I} 8$ atomes de carbone et de la proportion d'acide stéarique. Les acides saturés majeurs diminuent simultanément. Au cours de la période post-expérimentale on observe un retour progressif vers les proportions relatives observées au départ.

Simultanément à 1'augmentation des acides stéarique et monoène à 18 atomes de carbone, on observe une augmentation notable des acides diènes et triènes à I 8 atomes de carbone.

Tout ceci confirme les résultats déjà obtenus à diverses reprises antérieurement (cf. KuzDzaI-SAvore, Ig64). Quelques observations nouvelles peuvent être relevées en ce qui concerne les acides gras mineurs.

d) Les acides gras « mineurs».

${ }^{\circ}$ Les acides diènes conjugués.

I e taux des diènes conjugués du beurre varie nettement au cours de 1'expérimentation, par suite de la distribution du tourteau de lin (fig. 5). I)éterminé par spectrophotométrie dans 1'ultraviolet, ce taux varie de 0,4 à I,5 g p. Ioo g de matière grasse. La variation du taux des diènes conjugués est très régulière et s'aligne parfaitement sur la variation de la quantité de tourteau de lin distribuée. L'augmentation du taux de diènes conjugués se produit dans les 24 heures qui suivent la première distribution de tourteau gras, mais la diminution de ce taux après la suppression du tourteau de lin dans la ration est beaucoup plus lente : il faut attendre cinq jours environ après la dernière distribution de tourteau pour que le taux de diènes conjugués corresponde au taux de départ. 
TA

Proportions relatives

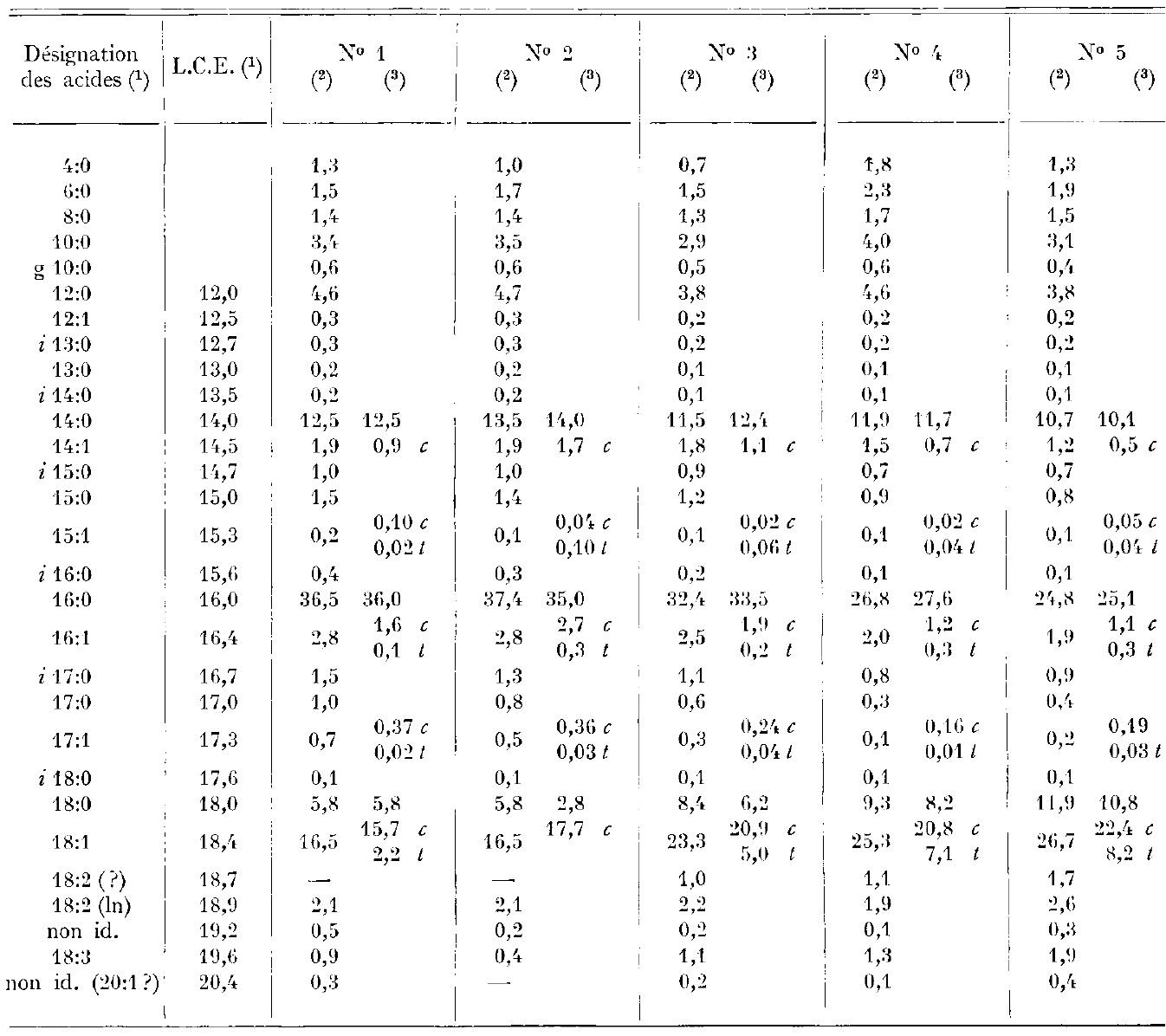

() Le premier chiffre est le nombre d'atomes de carbone. Le second est le nombre de doubles liaisons. c, cis; $t$, tra

(2) Résultats de l'analyse directe par chronatographie en phase gazeuse des esters méthyliques totaux. Résult

$\left({ }^{3}\right)$ Résultats de l'analyse par chromatographie en phase gazeuse des fractions antérieurement séparées par chron 


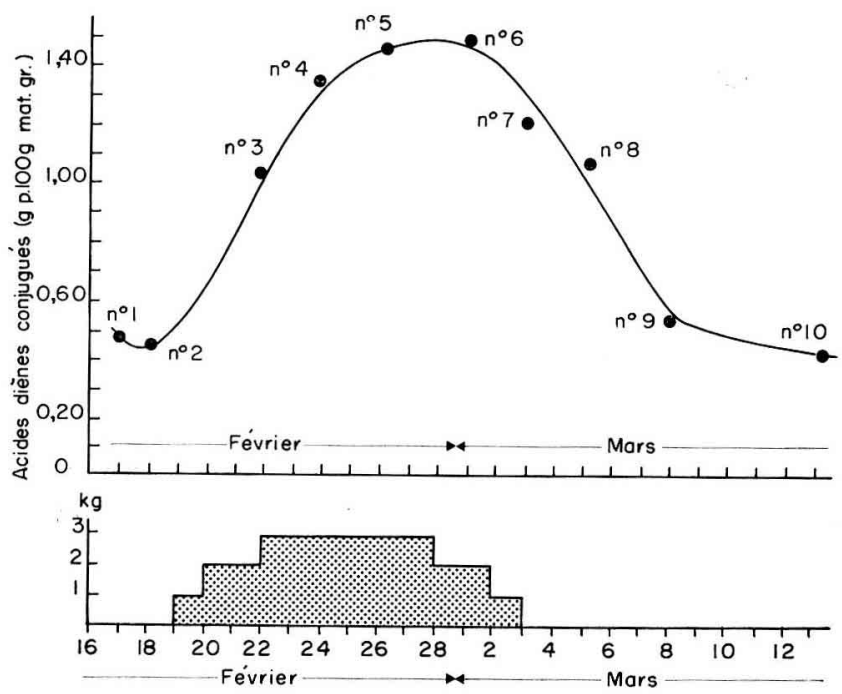

\section{1.. tourteau de lin}

Fig. 5. - Variation du taux d'acides diènes coniugués dans le beurre au cours de l'expérinenlation. (Résultats de l'analyse par spectrophotométrie dans l'ultra violet, selon BRICE et SwAIN, I945).
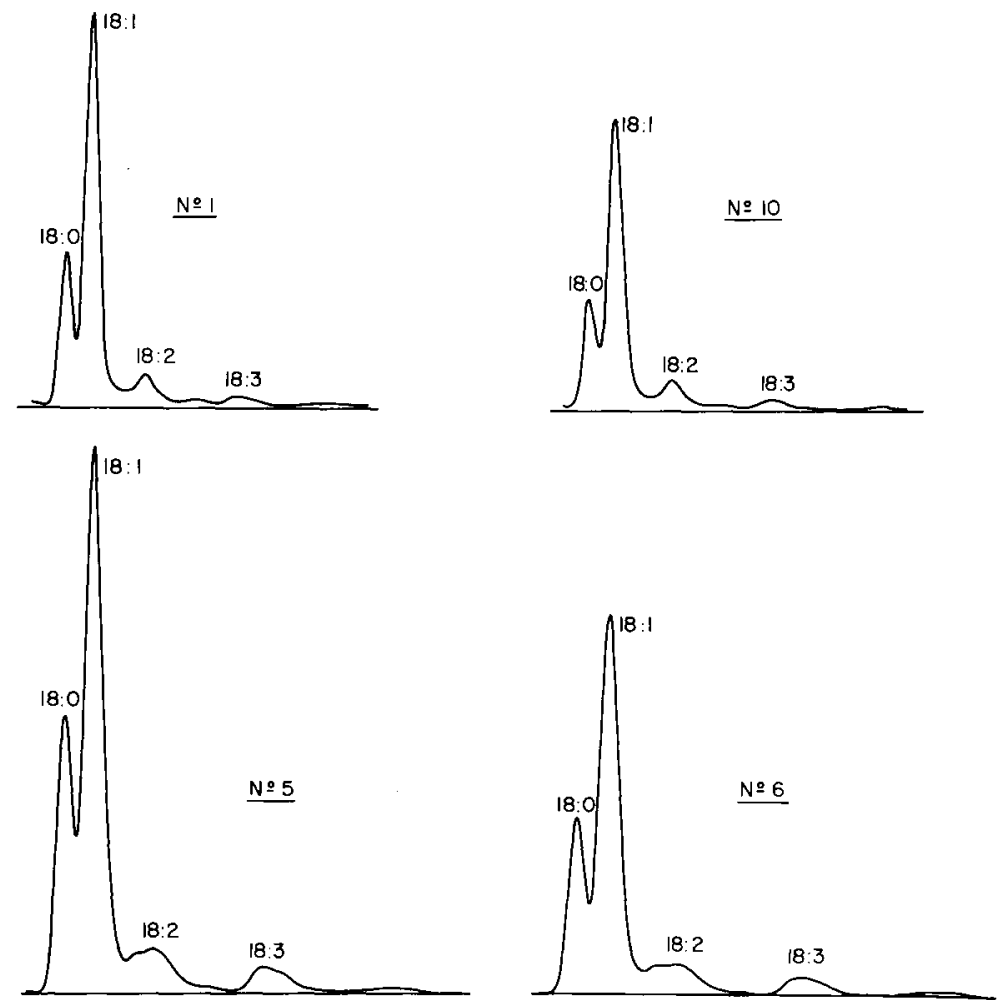

FIG. 6. - Etude comparée des chromatogrammes partiels (comprenant les pics: $18: 0,18: 1,18: 2$ et $18: 3$ ) des échantillons de beurre $n^{\mathrm{o}} 1$ et 10 (périodes pré-et post-expérimentales) el $n^{\mathrm{o}} 5$ et 6 (période expérimentale.) Conditions de l'analyse : Aerograph HyFi $6 \mathrm{co}$ (ionisation de flamme).

Colonne : $3048 \mathrm{~m}$ (Io pieds anglais) de long et $0,32 \mathrm{~cm}$ (1/8 de pouce anglais) de diamètre. Phase : DEGS à Io p. 10o $(\mathrm{p} / \mathrm{p})$ sur chromosorb W lavé à l'acide.

Température : $180^{\circ} \mathrm{C}$ environ.

Gaz vecteur : azote; débit : 25 à $30 \mathrm{ml} / \mathrm{minute}$. 
L'analyse par chromatographie en phase gazeuse des esters méthyliques totaux du beurre, n'a pas permis d'individualiser le ou les pics correspondant aux acides diènes conjugués. La proportion de ces acides n'apparaît donc pas dans le tableau 3 .

$2^{\circ}$ Particularité du pic «diène », 18:2.

Sur les chromatogrammes obtenus au cours de l'analyse directe des esters méthyliques totaux on observe que le pic correspondant aux diènes à 18 atomes de carbone présente une certaine particularité au cours de cette expérience, non seulement dans l'importance relative du pic des diènes mais dans la forme de ce pic. Ceci est montré sur la figure 6 . Alors que le pic des diènes est nettement séparé đu pic monoène pour les échantillons I et Io, cette séparation est moins nette sur les chromatogrammes correspondant aux échantillons 5 et 6 et l'on peut affirmer que ceci est dû̀ à 1'apparition d'un pic supplémentaire adjacent et antérieur au pic "vrai " du diène " linoléique ". Dans 1'évaluation quantitative des acides gras (tabl. 3) nous avons volontairement dissocié les deux parties du pic des "diènes ", et nous avons appelé " I 8:2, linoléique " le pic qui effectivement correspond à cet acide et " I8:2?" le pic antérieur visible surtout sur les chromatogrammes correspondant aux échantillons de beurre fabriqués au milieu de la période d'expérimentation.

On constate ainsi que la variation du taux d'acide linoléique est relativement faible au cours de l'expérimentation puisque les valeurs extrêmes observées sont I, 9 et 2,8 (en p. Ioo des esters totaux) alors que la variation atteignant le pic antérieur présente une plus grande amplitude: celle-ci correspond à une variation de taux allant des " traces " jusqu'à 2,0 p. Ioo (cf. tabl. 3).

Mais ceci n'implique pas que le pic antérieur au pic de l'acide linoléique vrai corresponde aux acides diènes conjugués ou à l'un des acides de ce groupe. Nous verrons pourquoi au cours de la discussion.

\section{$3^{\circ}$ Les acides monoènes mineurs du groupe trans.}

Nous avons indiqué que des acides monoènes à 15 , I6 et I7 atomes de carbone sont régulièrement présents dans les acides trans. L'isolement de ces acides et l'établissement de la configuration trans doit précéder l'affirmation qu'il s'agit vraiment d'acides trans. Or ceci n'a pas été effectué dans l'expérience présente. Néanmoins quelques estimations ont été faites sur ces acides monoènes mineurs du groupe trans.

Le taux de monoènes à I 6 atomes de carbone est en moyenne de 0,2 p. Ioo des acides totaux du beurre, avec des valeurs extrêmes de $0,09-0,27$, ce qui correspond à 6-20 p. Ioo de l'acide hexadécénoïque total.

L'acide monoène à $\mathrm{I} 5$ atomes de carbone présent dans les acides trans, représente 0,02 à $0,06 \mathrm{p}$. Ioo des acides totaux du beurre. S'il s'agissait vraiment du monoène trans, celui-ci représenterait une proportion relativement élevée de l'acide monoène à 15 atomes de carbone total (cf. tabl. 3).

La portion d'acide monoène à $\mathrm{I} 7$ atomes de carbone trouvée dans les acides trans est, par contre, toujours très faible représentant à peine Io p. Ioo de l'acide heptadécènoïque total.

$4^{\circ}$ Les autres acides mineurs.

On a représenté sur les figures 7 à Io les variations des proportions d'un certain nombre d'acides mineurs groupés selon leur nature : acides monoènes (fig. 7) acides 


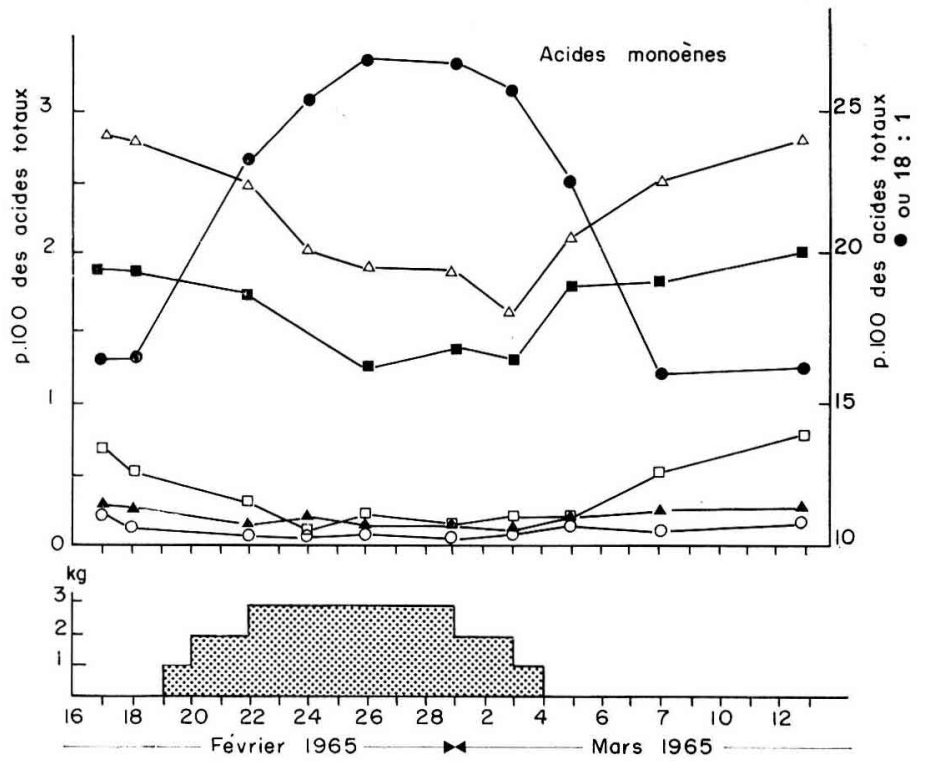

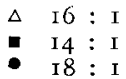

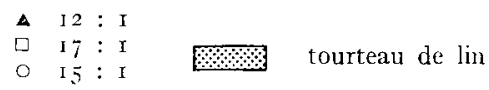

Frg. 7. - Varialion tu taux des acides monoènes dans le beurre au cours de l'expérimenlation (cf tabl. 3)

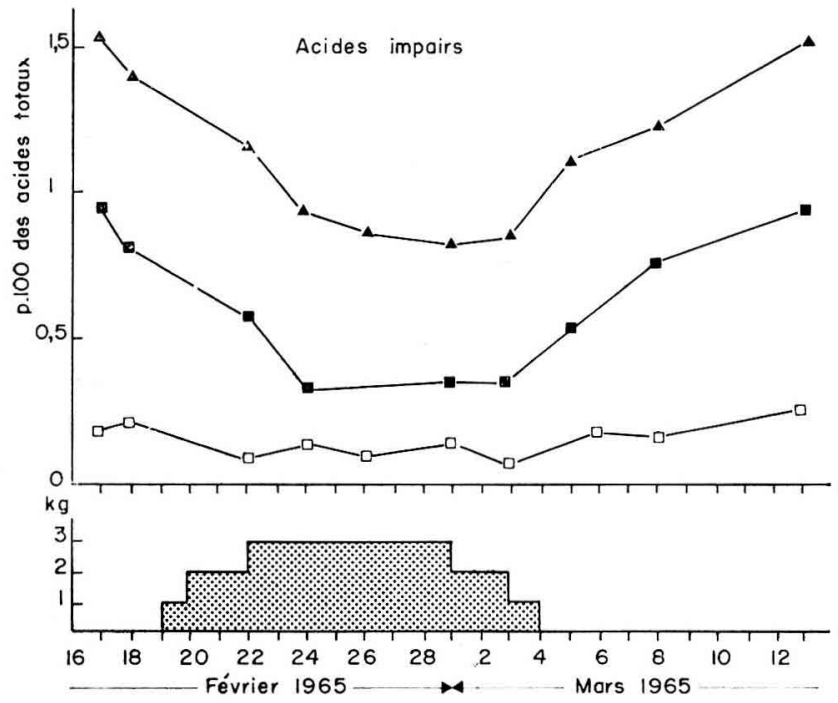

- I5:0

- $17: 0$

ㄷ I3:०

\$3 tourteau de lin

FIG. 8. - Variation du taux des acides à nombre impair d'atomes de carbone dans le beurre au cours de l'expérimentation (cf tabl. 3) 
- $i \mathrm{I} 7: 0$

$0 \quad i$ I5:0

A $i$ 16:0

$\square \quad i$ I4 :0

A $i$ : :0

tourteau
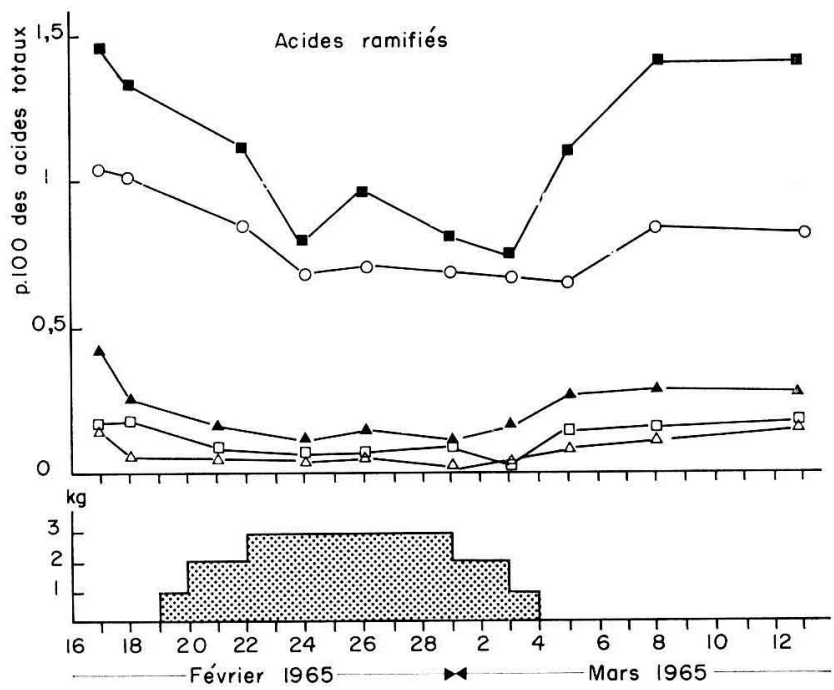

FIG. 9. - Variation du taux des acides ramifiés du beurre au cours de l'expérimentation (cf tabl. 3).

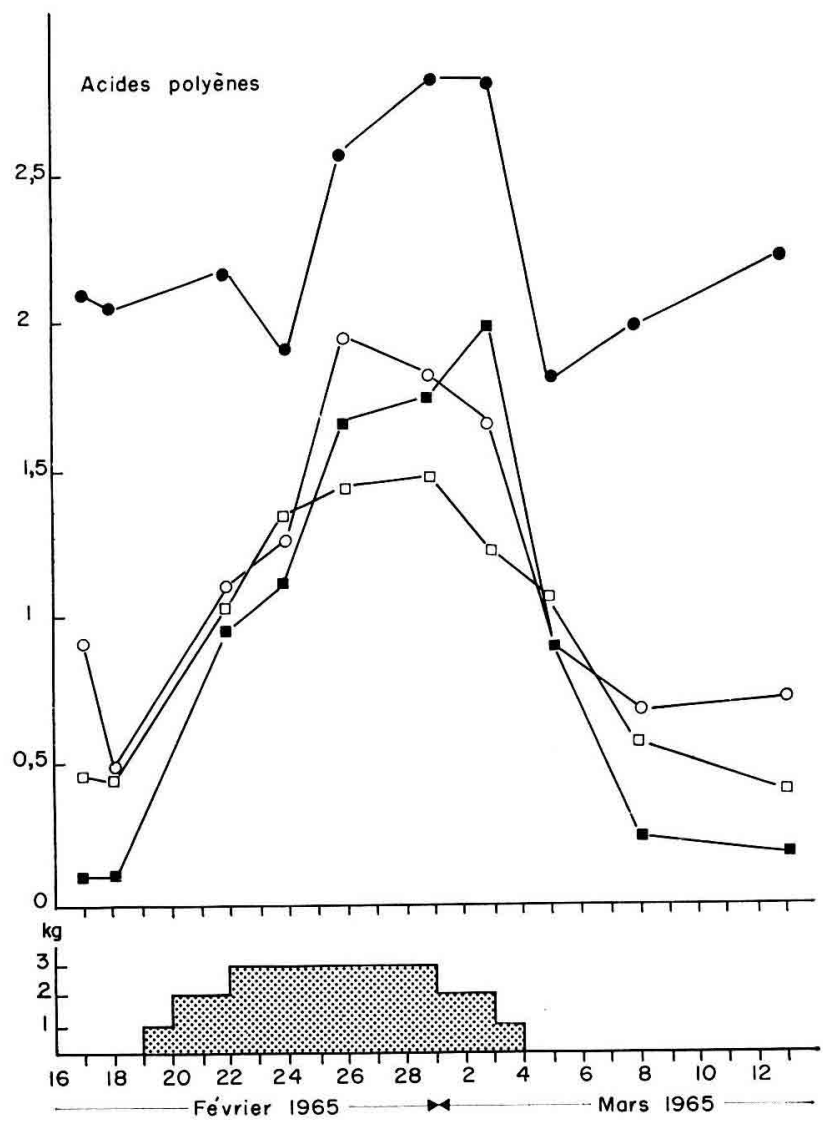

- $18: 2$ (linoléique) $\square$ I $8: 2$ (conj. U. V.)

O $18: 3$

- $18: 2$ (non linoléique)

FIG. 10. - Variation du taux des acides polyènes du beurre au cours de l'expérimentation (cf tabl. 3) 
à nombre impair d'atomes de carbone (fig. 8) acides ramifiés (fig. 9), acides polyènes (fig. Io).

On constate que d'une façon générale les proportions respectives de tous les acides à l'exception des acides à $I 8$ atomes de carbone, diminuent au cours de l'expérimentation et s'élèvent ensuite, et atteignent au bout d'une période post-expérimentale de deux semaines environ les proportions observées au départ.

Parmi les acides polyènes à IS atomes de carbone, l'acide linoléique s'élève relativement peu et d'une façon très temporaire. L'acide linolénique augmente au cours de l'expérimentation, et cette variation s'aligne sur celle de l'acide oléique.

\section{V. - DISCUSSION}

\section{A - Origine des acides trans monoènes}

Les acides trans monoènes à I 8 atomes de carbone constituent 90 p. Ioo des acides trans totaux, isolés et dosés selon le procédé analytique décrit. La variation du taux des acides trans du beurre, visible dans le tableau 2 est donc due essentiellement à la variation du taux des monoènes trans à I 8 atomes de carbone. La présence de ceux-ci dans les lipides du lait, est en relation directe avec la quantité d'acide linolénique présent dans la ration des vaches laitières. L'acide linolénique est donc le "précurseur " des acides trans monoènes à 18 atomes de carbone du beurre. Ce rôle de précurseur pourrait aussi être joué par l'acide linoléique, cet acide subissant aussi l'hydrogénation avec formation d'acides trans monoènes à $\mathrm{I} 8$ atomes de carbone dans le tractus digestif (ShORLAND et al., I957; WARD et al., I964).

Dans le cas de l'expérience présente l'acide linolénique constitue $60 \mathrm{p}$. Ioo des acides totaux de 1'huile de lin alors que l'acide linoléique n'en constitue que I7 p. Ioo La quantité d'acide linoléique introduite dans la ration n'en est pas moins notable. Cependant si l'addition à la ration d'une quantité importante d'acide linoléique entraîne une augmentation des monoènes à 18 atomes de carbone dans les lipides du lait (Hilditch et Williams, ig64; Matrisson et al., ig6i ; Kuzdzal-Savoie, I 064$)$, — sans qu'il soit possible de dire s'il y a ou non accroissement des monoènes

:- il n'y a pas d'accroissement simultané des acides diènes conjugués alors qu'un tel accroissement est toujours observé quand il y a un apport d'acide linolénique dans la ration (MATTSSON, I949; KuzDZAL-SAVOIE, Ig64). Dans le cas d'un apport d'acide linoléique dans la ration on observe généralement une augmentation faible mais sensible de l'acide linoléique dans le lait (MatTsson et al., ig6r ; Kuzdzat-Savore, x964). Dans 1'expérience poursuivie, on a effectivement observé une légère augmentation du taux d'acide linoléique (fig. Io), due sans doute à l'apport représenté par les 17 p. Ioo d'acide linoléique présent dans l'huile de lin. Mais la variation très importante du taux d'acides diènes conjugués est une preuve que le facteur responsable des variations atteignant la proportion d'acides monoènes trans est bien l'apport d'acide linolénique dans la ration.

\section{$\mathrm{B}$ - Variations de la teneur du beurre en acides trans}

Au cours de la période expérimentale, les vaches laitières ont reçu une quantité allant de I25 à $380 \mathrm{~g}$ d'acide linolénique par vache et par jour. Cette quantité d'acide 
linolénique est du même ordre que la quantité d'acide linolénique qui se trouve dans la ration des vaches laitières lorsque celles-ci séjournent au pâturage pendant la période de printemps (cf. Kuzdzal-SavoIE, I 964). L'alimentation de base n'apporte, dans 1'expérience présente, que 50 ou $60 \mathrm{~g}$ d'acide linolénique dans la ration journalière (cf. KuzdzaL-Savoie, 1964).

Les proportions d'acides diènes conjugués, d'acides trans, d'acide oléique et d'acide stéarique trouvées dans les échantillons de beurre fabriqués à partir du lait produit par les vaches en expérimentation sont aussi du même ordre que les proportions des mêmes composés observées dans le beurre commercial fabriqué au printemps ou à l'automne dans les régions où les vaches laitières séjournent alors au pâturage (cf. Kuzdzal-Savoie, I964; Kuzdzal-Savole et Raymond, I965).

On peut donc affirmer que, dans les conditions actuelles de vie et d'alimentation auxquelles les vaches sont soumises, les acides trans du beurre ne peuvent pas représenter une proportion supérieure à 7 ou $8 \mathrm{p}$. Ioo des acides gras totaux.

Ce taux pourrait-il s'élever si les conditions d'alimentation devenaient différentes? Si, par exemple, la distribution d'aliments concentrés de composition variée dans lesquels seraient incorporées des graisses hydrogénées, remplaçant alors pour une large part l'alimentation traditionnelle, devenait une pratique habituelle? S'il est démontré que les acides trans (qui constituent une fraction majeure des acides gras des graisses hydrogénées) peuvent "passer " dans le lait, - comme cela a été prouvé pour d'autres acides gras (GLAscock et al., I955; KuZDZAL-SAvoIE et PAQUOT, I962) - , le taux des acides trans du lait pourrait alors dépasser le taux maximum que nous venons d'indiquer. Ce point sera précisé dans une prochaine expérimentation.

\section{C - Nature des acides trans}

L'étude des acides trans que nous venons de présenter ne donne aucune information sur la nature exacte des acides trans à l'exception du nombre d'atomes de carbone.

Les proportions relatives des différents isomères de position seront déterminées ultérieurement afin de préciser si les acides monoènes trans à I 8 atomes de carbone isolés et dosés dans l'étude présente possèdent la double liaison préférentiellement sur le carbone i r. En effet, dans leur étude sur l'hydrogénation dans le rumen (hydrogénation portant sur l'acide linolénique), WARD et al. (I964) ont montré que les emplacements préférentiels de la double liaison des monoènes formés étaient les atomes de carbone 13 et I4 ; les emplacements sur les atomes I6, I I et io ne représentant respectivement que 6, i 2 et $6 \mathrm{p}$. Ioo des cas. Cependant les auteurs cités n'ont pas déterminé les emplacements préférentiels de la double liaison des seuls monoènes trans. Il serait souhaitable de rechercher si les monoènes trans formés au niveau du rumen possèdent préférentiellement la double liaison sur le carbone II. Ceci permettrait de situer exactement l'origine des acides trans prédominants du lait.

\section{$\mathrm{D}$ - Origine et nature des acides trans autres que les monoènes à 18 atomes de carbone}

Nous avons régulièrement observé au cours de cette étude la présence d"un acide monoène à $\mathrm{I} 6$ atomes de carbone dans le groupe des acides trans. L'acide trans-hexadécénoïque a été précédemment signalé dans le beurre (SMITH et al., I954; BACK- 
DERF et BROWN, I958; HERB et al., I962). Selon les auteurs il représente environ $20 \mathrm{p}$. Ioo de l'acide hexadécènoïque total. Dans l'expérience décrite nous avons trouvé que l'acide hexadécènoïque accompagnant les acides trans représente une proportion de 6 à $20 \mathrm{p}$. Ioo de l'acide hexadécènoïque total. Cette similitude dans les proportions appuie l'hypothèse selon laquelle l'acide hexadécènoïque trouvé dans les acides trans serait l'acide trans hexadécènoïque.

En ce qui concerne 1'acide monoène à $I 7$ atomes de carbone présent dans les acides trans, l'interprétation est plus difficile. En effet, Hansen et al. (I960) ont établi que l'acide heptadécènoïque du beurre possédait la seule configuration cis. La faible fraction (ro p. IOo) d'acide heptadécènoïque que nous avons trouvée dans les acides trans ne constituerait-elle pas une impureté?

Nous avons écrit au début de cette étude que les aliments généralement distribués aux vaches laitières ne renfermaient pas d'acides trans. Cette absence n'est cependant pas absolue. Ainsi WEEnink et SHORLAND (I964) ont isolé récemment un acide trans à $\mathbf{6} 6$ atomes de carbone dans les lipides des feuilles de trèfle. Cet acide constitue $\mathrm{I}, 4$ p. Ioo des acides totaux ; il existe également dans les lipides du ray-grass (Lolium perenne) et d'autres végétaux.

Si l'existence de certains acides trans mineurs se trouvait confirmée dans les lipides du lait. leur origine alimentaire pourrait donc être aussi envisagée.

\section{$\mathrm{E}$ - Les acides diènes conjugués}

Le taux de diènes conjugués (évalué par spectrophotométrie ultraviolette) s'est élevé au cours de l'expérience décrite de 0,4 à I,5 p. Ioo des acides totaux.

Une telle modification de pourcentage devrait correspondre à une modification visible sur les chromatogrammes.

Si l'on se réfère aux travaux de Magidman et al. (i962), de Lichteield et al. (1963), de WARD et al. (I964) et de HostetTtER et al. (I965) on peut affirmer que, sur colonne polaire (succinate de diéthylène glycol, par exemple) l'acide conjugué cistrans apparaît pratiquement en même temps que l'acide linolénique et l'acide conjugué trans-trans un peu plus loin, l'acide conjugué cis-cis occupant une position intermédiaire.

Actuellement il est admis que l'acide diène conjugué du beurre est principalement cis-trans et secondairement trans-trans (SMITH et JACK, I954).

Cependant parmi les composés intermédiaires formés au cours de l'hydrogénation dans le rumen à partir d'acide linolénique, WARD et al. (1964) signalent un acide diène conjugué cis-cis. La possibilité d'une absorption à travers la paroi du rumen ayant été évoquée (McCARTHy, I962), il se pourrait que des traces d'acides conjugués cis-cis existent dans le beurre.

Quoi qu'il en soit une élévation du taux de diènes conjugués doit correspondre sur les chromatogrammes à un accroissement du pic "linolénique " ou d'un pic plus lointain.

En fait on observe bien un accroissement du pic attribué à l'acide linolénique (cf. tab1. 3). Mais la distribution d'huile de lin entraîne normalement une augmentation de 1'acide linolénique lui-même (KuzDzal-Savore, I964). Cet accroissement est, dans l'expérience présente, cependant plus fort que l'accroissement auquel on aurait pu s'attendre compte tenu des résultats antérieurs (cf. KuzDzar-SAvore, 
I964). Il est donc possible qu'une partie de l'acide diène conjugué soit intégrée dans le pic «linolénique ».

$$
\text { F - Etude du pic 18:2 }
$$

Le pic correspondant aux diènes non conjugués (acide linoléique) aurait dû n'être que peu modifié puisque l'on sait que l'addition d'huile de lin à la ration n'entraîne pratiquement pas d'augmentation des diènes non conjugués (KuzDzalSavore, I 964). Or, on constate un accroissement net du pic " linoléique " et son dédoublement (c. fig. 6). A quel acide attribuer le pic antérieur observé ?

Le déplacement de la double liaison le long de la chaîne modifie aussi le temps de rétention sur phase polaire telle que succinate de diéthylène glycol : celui-ci est d'autant plus grand que la distance entre le groupe carboxyle et la double liaison est plus grande (ceci est valable pour les acides monoènes comme pour les acides diènes à $I 8$ atomes de carbone), ou que (dans le cas des diènes) la distance entre deux doubles liaisons s'accroît (HOSTETTER et al., I965).

Donc, aucun acide diène, parmi ceux signalés dans le beurre, ne peut se placer avant le pic correspondant à l'acide linoléique, dans les conditions de chromatographie, décrites ici. Ce pic pourrait donc être dû à un acide monoène à $\mathrm{r} 8$ atomes de carbone dont la double liaison serait placée très loin du carboxyle. L'acide trans, I6-octadécénoïque correspond à cette définition. Cet acide constituerait, selon BACKDERF et BRown (I958), i à 2 p. Ioo des acides totaux du beurre, et, selon HanSEN et SHORLAND (1963) seulement 0,2 p. IOO. La variation observée dans l'expérience décrite $\mathrm{du}$ pourcentage que représente le " pic antérieur " à l'acide linoléique (désigné par " I 8:2 ? non linoléique 》 sur la figure Io) est du même ordre de grandeur.

Si l'hypothèse avancée est exacte nous devons retrouver cet acide monoène trans dans le groupe " acides trans" obtenu par chromatographie sur colonne de silicagel imprégné de nitrate d'argent. L'image des acides trans, révèle toujours la présence d'un épaulement sur la ligne descendante du pic monoène trans. Nous avions précisément attribué cet épaulement à l'acide trans, r6-octadécénoïque (KUZDZALSavore et RAYMOND, I965).

Cependant l'isolement de cet acide et son identification sont nécessaires pour être définitivement fixés sur ce point.

Reçu pour publication en mars 1966.

\section{REMERCIEMENTS}

Ce travail a bénéficié d'une subvention du Comité de Nutrition de la Délégation générale à la Recherche scientifique à qui nous adressons nos remerciements.

D'autre part nous exprimons notre gratitude à M. MOCQUot, directeur de la Station centrale de Recherches laitières et de Technologie des Produits animaux et à M. PAQuoT, directeur du Laboratoire de Lipochimie (Centre national de Recherche scientifique) qui nous ont aidés et conseillés au cours de ce travail.

Nous remercions également M. Journet, et M. Marquis de la Station de Recherches sur l'Élevage (Centre national de Recherches zootechniques) dont la collaboration nous a permis de mener à bien l'expérience décrite. 


\title{
SUMMARY
}

\author{
THE " TRANS " FATTY ACIDS OF BUTTER. 2, CONTRIBUTION TO TIL STUDY \\ OF TIIEIR ORIGIN
}

The trans fatty acids of butter are already present in the lipids of the milk. Their origins essentially endogenous, for the natural feeds habitually given to milking cows contain only a negligeable amount of trans fatty acids. Various earlier studies have shown that trans fatty acids in milk increase as the amount of young herbage in the ration of the cow increases. The trans fatty acids of the lipids of milk are essentially the result of hydrogenation in the rumen of the polyunsaturated acids in the ration. Now, linolenic acid is the main acid in the lipids of herbage and green fodder. Its proportion in the lipids of the ration then varies widely in the course of the year.

The object of the study described was to find if there is a direct relation between the linolenic acid of the ration and the content of trans fatty acids in the milk or butter.

The experiment was with 3 French Friesian cows belonging to the herd of the Centre national de Recherches zootechniques at Jouy-en-Josas. These cows were given, during the period of winter feeding in stalls, a supplement of unextracted linseed meal in amouts increasing, then decreasing. The maximum daily amount given, $3 \mathrm{~kg}$, was equivalent to $380 \mathrm{~g}$ linolenic acid. During the experiment which lasted two weeks, ten samples of butter were prepared.

The relative proportions of the major fatty acids and about twenty minor fatty acids were estimated by gas chromatography of the total methyl esters. The trans fatty acids were isolated by column chromatography and thin-layer chromatography on silica gel impregnated with silver nitrate and weighed.

The conjugated diene fatty acids were estimated by ultraviolet spectrophotometry.

The linseed meal caused a marked increase in the amount of fat secreted by the cows.

As regards the major fatty acids there was an increase in the contents of stearic acid and monoene acids with 18 carbon atoms and a decrease in saturated acids with $\mathrm{I} 6$ and $\mathrm{I} 4$ carbon atoms.

The changes in the contents of trans and conjugated diene fatty acids were parallel to the changes in monoene acids with 18 carbon atoms. There was thus a direct relation between the linolenic acid content of the diet of the cows and the content of total trans fatty acids in the milk.

As regards the minor acids, the respective contents of saturated acids with an uneven number of carbon atoms, of branched-chain acids and of monoene acids other than $18:$ i fell during the time that unextracted linseed meal was given.

Inclusion of linseed oil in the diet thus allows a fairly true reproduction of certain changes in the relative proportions of fatty acids wich are seen during the course of the year.

\section{RÉFÉRENCES BIBLIOGRAPHIQUES}

BACKDERF I. R., BROWN J. B., I95\%. Further contribution to the nature of the monoethenoic fatty acids of butterfat. Arch. Bioch. Biophys., 76, I 5 .

IBRICE B. A., SWAIN M. L., I945. Eltraviolet absorption method for the determination of polyunsaturated constituents in fatty materials. J. Optical Soc. Amer., 35, $35^{2}$.

BroUwer Ji., JONKER-SChEFFEnER M. C. E., I 946 . Vaccenic acid in butterfat and the fluctuations in its amount. Rec. Traw. Chim. Pays-Bas, 65, 408.

Dutron H. J., I963. Kinetics of linolenate hydrogenation. J. Amer. Oil Chem. Soc., 40, 35.

Glascock R.F., Duncombe W. G., Reinies I.. R., i956. Studies on the origin of milk fat. 2 . The secretion of dietary long chain fatty acids in milk fat by ruminants. Biochem. J., 8., 535 .

Hansen R. P., Shorland F. B., CoOkE N. J., 1960. The isolation of cis-9-heptadecenoic acid from butterfat. Biochem. J., 9ry, 64.

Hansen R. P., Shorland F. B., Cooke N. J., I959. Isolation and identification of the high molecular weight saturated fatty acids of butterfat. J.Dairy Res., 26, I9o.

Hartman I., Shorl.and F. B., MCDonald I. R. C., 1954. Occurrence of trans acids in animal fats. Nature (London), 174, I85.

Hartman I., Shorland F. B., Moir R., 1956. Occurrence of trans unsaturated fatty acids in horse faeces. Nalure (Iondon), 178, 1057. 
Herb S. F., Magidman F., Luddy F. R., Riemenschneider R. W., i962. Fatty acids of cow's milk. 13. Composition by gas-liquid chromatography aided by other methods of fractionation. J. Amer. Oil C'hem. Soc., 39, I42.

Hilditci T. B., Williams P. N., I964. The chemical constitution of natural fats. 4th ed. Chapman et Hall, Londres.

Hostetter H. H., SeN N., Holman R. T., I965. Characterization on unsaturated fatty acids by gasliquid chromatography. J. Amer. Oil Chem. Soc., 42, 537.

IVERSON J. L., EISNER J., lirestone D., 1965. Detection of trace fatty acids in fats and oils by urea fractionation and gas-liquid chromatography. J. Amer. Oil Chem. Soc., 42, 1063.

KATZ I., KEENEY M., I 963 . Location of the double bonds in the octadecenoic acid fractions of milk fat, beef fat and lard. J. Dairy Sci., 46, 605.

KvzDzal-Savoie S., i964. Influence de la composition de la ration sur la composition chimique du beurre de vache. Thèse (série A, $n^{0} 4277^{-5128), ~ l i a c . ~ S c i ., ~ P a r i s . ~}$

Kr'zDZAL-Savole S., KuzDzal W., I 961. Influence de la mise à l'herbe des vaches laitières sur les indices de la matière grasse du beurre et sur les teneurs en différents acides gras polyinsaturés. Ann. Biol. anim. Bioch. Biophys., 1, 47.

KuzDzal-Savoie S., KuzDzal W., 1962. Variations observées dans les proportions relatives des acides gras du lait de vache pendant la période de mise à l'herbe. XVIe Cong. Int. Lait., Copenhague, 1, 270.

Kuzuzal-Savoie S., Paquot C., I 662 . Chromatographie en phase gazeuse et lipochimie. 3. Identification d'un beurre de constitution anormale. Ann. Fals. Exp. Chim., 637, 9 et Oléagineux, 17, 185 .

Kuzizal-Savoie S., Raymond J., i965. Les acides gras trans du beurre. I. Isolement et dosage. Ann. Biol. anim. Bioch. Biophys., 5, 497.

Litchfikid C., Isbeli A. F., Reiser R., Ig62. Analysis of the geometric isomers of methyl linoleate by Eas-chromatography. J. Amer. Oil Chem. Soc., 39, $33 \mathrm{I}$.

LitchfieLd C., Reiser R., IsBei.L A. E., 1963. The analysis of cis-trans fatty acid isomers using gas-liquid ihromatography. J. Amer. Oil. Chem. Soc., 40, 302.

McCartily R. D., i962. Cité par Ward F. V., Scott T. W., Dawson R. M. C., ig64.

Magidmax P., Herb S. F., Barford R. A., Riemenschneider R. W., 1962. Fatty acids of cow's milk. A. 'Techniques employed in supplementing gas-liquid chromatography for identification of fatty acids. J. Amer. Oil Chem. Soc., 39, 137.

Matssox S., i949. Polyunsaturated fatty acids in butter and their influence on the oxidation of butter. I. Methods of analysis and preliminary results. Medd. Slatens Mejeriforsok, 26, 29.

Mattsson S., Thone K. E., Swartling P., i95I. Polyunsaturated fatty acids in butter and their influence on the oxidation of butter. 2. Oxidation of butterfat of different composition. Medd. Statens M ejeriforsok, 33, I.

O'J.EARY W. M., I959. Studies of the utilization of $\mathrm{C}^{14}$ labeled octadecenoic acids by Laclobacillus arabinosus. 7. Bacteriol., $7 \mathrm{y}, 367$.

Pallota V., Losi G., Zorsut C., 1965. Sulla determinazione dell'acido elaidinico negli olii di crucifere. Riv. ilal. sostanse grasse, $\mathbf{4 2}$, $\mathbf{1}^{2}$.

Shorland F. B., WeEnink R. O., Johns A. T., McDonald I. R. C., I957. The effect of sheep rumen contents on unsaturated fatty acids. Biochem. J., 67, $32 \%$.

SMIti L. M., JACK E., I954. The unsaturated fatty acids of milk fat. II. Conjugated and non-conjugated constituents. J. Dairy Sci, 37, 390.

Surtir L. M., Freeman N. K., JACK E., I 954. The unsaturated fatty acids of milk fat. III. Geometrical isomerism. J. Dairy Sci., 37, 399.

Sreenivasan B., Nowakowska J., Jones E. P., Selke F., Scholfield C. R., Dutton H. J., I963. Hydrogenation of linolenate. VII. Separation and identification of isomeric dienes and monoenes. J. Amer. Oil Chem. Soc., 40, 45.

Ward P. F. V., SCOTT T. W., Dawson R. M. C., I964. The hydrogenation of unsaturated fatty acids in the ovine digestive tract. Biochem. J., 92, 60.

WEENINK R. O., SHORLAND F. B., I964. The isolation of trans-3-hexadecenoic acid from the lipids of red-clover (Trifolium pralense) leaves. Biochim. Biophys. Acla, 84, 6r3. 